EPJ manuscript No.

(will be inserted by the editor)

\title{
Backward pion-nucleon scattering
}

\author{
F. Huang ${ }^{1}$, A. Sibirtsev ${ }^{2,3}$, J. Haidenbauer ${ }^{4,5}$, S. Krewald ${ }^{4,5}$ and U.-G. Meißner ${ }^{2,4,5}$ \\ 1 Department of Physics and Astronomy, The University of Georgia, Athens, Georgia 30602, USA \\ 2 Helmholtz-Institut für Strahlen- und Kernphysik (Theorie) und Bethe Center for Theoretical Physics, Universität Bonn, \\ D-53115 Bonn, Germany \\ ${ }^{3}$ Excited Baryon Analysis Center (EBAC), Thomas Jefferson National Accelerator Facility, Newport News, Virginia 23606, \\ USA \\ ${ }^{4}$ Institut für Kernphysik and Jülich Center for Hadron Physics, Forschungszentrum Jülich, D-52425 Jülich, Germany \\ ${ }^{5}$ Institute of Advanced Simulation, Forschungszentrum Jülich, D-52425 Jülich, Germany
}

Received: date / Revised version: date

\begin{abstract}
A global analysis of the world data on differential cross sections and polarization asymmetries of backward pion-nucleon scattering for invariant collision energies above $3 \mathrm{GeV}$ is performed in a Regge model. Including the $N_{\alpha}, N_{\gamma}, \Delta_{\delta}$ and $\Delta_{\beta}$ trajectories, we reproduce both angular distributions and polarization data for small values of the Mandelstam variable $u$, in contrast to previous analyses. The model amplitude is used to obtain evidence for baryon resonances with mass below $3 \mathrm{GeV}$. Our analysis suggests a $G_{39}$ resonance with a mass of $2.83 \mathrm{GeV}$ as member of the $\Delta_{\beta}$ trajectory from the corresponding Chew-Frautschi plot.
\end{abstract}

PACS. 13.75.-n Hadron-induced low- and intermediate energy reactions - 14.20.Gk Baryon resonances with $\mathrm{S}=0-11.55$.Jy Regge formalism

\section{Introduction}

The excitation spectrum of the nucleon in the energy range up to $2.5 \mathrm{GeV}$ is presently investigated with electromagnetically induced reactions at JLab, ELSA and Mainz. A major progress is expected from the experimental determination of polarization observables and exclusive measurements. New theoretical methods appear to be necessary for the analysis of the incoming data, as traditional partial wave analyses have to deal with a large number of parameters. For instance, the methods developed at EBAC at JLab are based on theories of non-resonant meson dynamics which, taken together with the resonant contributions, describe a variety of reactions. However at large energies, two-body reactions show regular features which with very high likelihood are not due to individual resonances. For energies above $2 \mathrm{GeV}$, the angular distributions are strongly forward peaked and show a smooth energy dependence. Moreover, at backward angles there is another regularity. In that angular region many reactions show a rise of the cross sections, with a magnitude that depends smoothly on the energy.

Most of our knowledge about resonances with large spin has been obtained from the study of pion scattering near $180^{\circ}$. A summary of models for high energy mesonnucleon backward scattering is given in refs. $1,2,3,4,5$. These models are based on Regge phenomenology using parameters determined by a systematic analysis of the backward differential cross section at different energies that were available before 1972. None of these models can describe simultaneously the data on differential cross sections and polarizations available at different energies. Experimental results at certain fixed energies were reproduced by introducing non-Regge terms 6] in addition to the standard Regge amplitudes. There are data for $\pi N$ backward scattering published after 1974 [7, 8, 9, 10,11, but unfortunately, most of these data have never been analyzed in the framework of the previous Regge models.

A major goal of this work is to analyze differential cross sections and polarization data of $\pi N$ backward scattering for invariant collision energy $\sqrt{s} \geq 3 \mathrm{GeV}$. We include experimental results available for the reactions $\pi^{+} p \rightarrow$ $\pi^{+} p, \pi^{-} p \rightarrow \pi^{-} p$ and $\pi^{-} p \rightarrow \pi^{0} n$. Regge phenomenology is applied in order to fix the reaction amplitude given by the contribution from four exchange trajectories, namely $N_{\alpha}, N_{\gamma}, \Delta_{\delta}$ and $\Delta_{\beta}$. These trajectories are parameterized by real linear functions of the squared four-momentum transfer $u$. Using 505 data points, we obtain a $\chi^{2}$ per data point of 1.84 .

Let us briefly recall the status of the published Regge phenomenology for backward pion-nucleon scattering. The differential cross section data for the reactions $\pi^{+} p \rightarrow$ $\pi^{+} p, \pi^{-} p \rightarrow \pi^{-} p$, and $\pi^{-} p \rightarrow \pi^{0} n$ can be described by three baryon trajectories, called $N_{\alpha}, N_{\gamma}$ and $\Delta_{\delta}$, which start with the nucleon, the $D_{13}(1520)$, and the $\Delta_{33}(1232)$, respectively. The $N_{\alpha}$ and $\Delta_{\delta}$ trajectories are the leading 
baryon trajectories for the $u$-channel isospin $I_{u}=1 / 2$ and $I_{u}=3 / 2$ reactions. The dip structure at $u \approx-0.15 \mathrm{GeV}^{2}$ in the backward $\pi^{+} p \rightarrow \pi^{+} p$ differential cross section is due to a zero in the $N_{\alpha}$ trajectory. A third trajectory $N_{\gamma}$ is needed because of the differences in the dip structure of the $\pi^{+} p \rightarrow \pi^{+} p$ and $\pi^{-} p \rightarrow \pi^{0} n$ differential cross sections. First polarization data were obtained after 1971 [12, 13, 14, 15. None of the Regge models available at that time could describe those data. Furthermore, for the $\pi^{-} p \rightarrow \pi^{-} p$ reaction the $N_{\alpha}$ and $N_{\gamma}$ exchanges do not contribute. Thus, within the frame of pole-exchange Regge models there is no relative phase between the spin flip and spin nonflip amplitudes from the $\Delta_{\delta}$ trajectory alone. As a consequence, the available models predicted zero polarization for the $\pi^{-} p \rightarrow \pi^{-} \mathrm{p}$ reaction, in contradiction to the polarization data.

Several attempts were made in order to resolve the conflict between polarization data and the models. First, let us mention the Regge model of ref. [4] which originally included only the $N_{\alpha}$ and $\Delta_{\delta}$ trajectories. The polarization predicted by this model for the $\pi^{-} p \rightarrow \pi^{-} p$ reaction was not zero because the trajectories were considered as complex functions parameterized in a quite sophisticated way. Later on it was found that the predictions of this model disagreed with the polarization data and the model was substantially modified 16 through additional inclusion of the $N_{\gamma}$ trajectory and by using sophisticated parametrizations of the vertex functions. After that the differential cross section and polarization at two beam momenta, 5.91 and $6 \mathrm{GeV}$, were well fitted. It was unclear whether this modified model still is able to reproduce data on differential cross sections available at other energies that were analyzed originally 4, because the systematic analysis was not repeated.

In the Regge analysis of ref. [17] the $N_{\alpha}$ and $N_{\gamma}$ trajectories were taken as real functions. But, following refs. [5, 18 the $\Delta_{\delta}$ contribution was parameterized differently with regard to the real and imaginary part of the amplitude. That allows to obtain relative phases between the spin flip and non-flip amplitudes as required by the non-zero polarization for the $\pi^{-} p \rightarrow \pi^{-} p$ process. It was shown that this model reproduces well the data on differential cross sections at pion momenta above $23 \mathrm{GeV}$ and the $\pi^{+} p \rightarrow \pi^{+} p$ polarization [12. However, these calculations could not describe the polarization data [14] in the $\pi^{-} p \rightarrow \pi^{-} p$ reaction. Although a reasonable description of the data was achieved, except for the $\pi^{-} p$ polarization, one should note that the modification of the $\Delta_{\delta}$ amplitude employed is not conventional in Regge phenomenology. Furthermore, it was not shown whether this model can reproduce the data at momenta below $23 \mathrm{GeV}$.

Another effort to describe the data on differential cross sections and polarizations was presented in ref. [6]. Only data at the pion momentum of $3.5 \mathrm{GeV}$ [15, 19,20, 21, 22 and $6 \mathrm{GeV}$ [13, 14,23,24] were included in the analysis performed with two different Regge models. The first one takes into account the $N_{\alpha}, N_{\gamma}$ and the modified [5, 18] $\Delta_{\delta}$ contributions discussed above. The second model [25] accounts for amplitudes given by the standard $N_{\alpha}, N_{\gamma}$ and
$\Delta_{\delta}$ Regge pole terms, but includes in addition a coherent background amplitude which is ascribed to quark rearrangement processes [26]. It was shown that both models reproduce the data well when fitted separately to the experimental results at momenta of $3.5 \mathrm{GeV}$ and $6 \mathrm{GeV}$. But it was not possible to obtain a reasonable description of the experiments within a simultaneous fit of the $3.5 \mathrm{GeV}$ and $6 \mathrm{GeV}$ data. Therefore, it was speculated that the polarization data for the $\pi^{-} p \rightarrow \pi^{-} p$ reaction might indicate that some of the amplitudes have an energy dependence that differs from the power law in invariant collision energy, which is typical for Regge phenomenology. However, any definite conclusion requires more systematic and comprehensive theoretical studies of backward pion scattering, which was not done.

The present study shows that the addition of a second Delta trajectory leads to an economic description of backward pion-nucleon scattering for large energies in terms of a simple Regge phenomenology. The amplitudes obtained at high energies allow an extrapolation to lower energies. We inspect how rapidly the Regge phenomenology starts to deviate from the data at invariant collision energies $2.4 \leq \sqrt{s} \leq 3 \mathrm{GeV}$. The analysis concentrates on available polarization data that are expected to be sensitive to possible contributions of high mass resonances. The indicated energy range is chosen for two principal reasons. First, the energy dependence of the differential cross section for the pion-nucleon scattering at $180^{\circ}$ shows some structures at these energies. Second, many earlier analyses [27, 28, 29, 30. found evidence for excited baryons with masses within this energy range. We study in detail the data available for $\pi^{+} p \rightarrow \pi^{+} p, \pi^{-} p \rightarrow \pi^{-} p$ and $\pi^{-} p \rightarrow \pi^{0} n$ scattering at $180^{\circ}$ and calculate the confidence level for the discrepancy between the data and our results. That allows us to estimate whether the oscillations of differential cross section around the continuation of the Regge result is of systematic or rather of statistical nature.

Finally we investigate the relation between the baryon trajectories fixed by our analysis in the scattering region and the baryon spectrum. Indeed the ordering of the hadronic states according to the Regge trajectories in the Chew-Frautschi plot is one of the most remarkable features of the Regge phenomenology. However, the Regge classification of baryons in many studies [31,32, 33 , 34 was done by using known or predicted states and not scattering data. Here we address the question whether the trajectories obtained in the analysis of backward pion scattering are the same as those given by the known baryon spectrum.

The paper is organized as follows. In sect. 2, we introduce our formalism. Sect. 3 provides a comparison of the results of our calculations with data on differential cross sections and polarizations at invariant collision energies above $3 \mathrm{GeV}$. In sect. 4 the amplitude is extrapolated to lower energies. The paper ends with a Summary. An appendix summarizes the available world data set on backward pion-nucleon scattering. 


\section{Formalism}

The differential cross section for backward pion-nucleon scattering reads

$$
\frac{d \sigma}{d u}=\frac{\left|\mathcal{M}^{++}\right|^{2}+\left|\mathcal{M}^{+-}\right|^{2}}{64 \pi s q^{2}} .
$$

Here, the $s$-channel helicity non-flip and flip amplitudes are called $\mathcal{M}^{++}$and $\mathcal{M}^{+-}$, respectively, while $q$ denotes the pion momentum in the $s$-channel center-of-mass (CM) system, using the notations $\mathrm{s}, \mathrm{t}$, and $\mathrm{u}$ for the Mandelstam variables. The polarization asymmetry is given by

$$
P=\frac{2 \operatorname{Im}\left[\mathcal{M}^{++} \mathcal{M}^{+-*}\right]}{\left|\mathcal{M}^{++}\right|^{2}+\left|\mathcal{M}^{+-}\right|^{2}} .
$$

Taking the amplitudes with specified $u$-channel isospin, we can write the $\pi N$ backward scattering amplitudes as

$$
\begin{aligned}
\mathcal{M}^{\pi^{+} p \rightarrow \pi^{+} p} & =\frac{2}{3} \mathcal{M}^{N}+\frac{1}{3} \mathcal{M}^{\Delta}, \\
\mathcal{M}^{\pi^{-} p \rightarrow \pi^{-} p} & =\mathcal{M}^{\Delta}, \\
\mathcal{M}^{\pi^{-} p \rightarrow \pi^{0} n} & =\frac{\sqrt{2}}{3} \mathcal{M}^{N}-\frac{\sqrt{2}}{3} \mathcal{M}^{\Delta},
\end{aligned}
$$

where the $N$ and $\Delta$ superscripts denote $u$-channel isospin $1 / 2$ and $3 / 2$ contributions, respectively. The $s$-channel helicity amplitudes are expressed in terms of the invariant amplitudes $A$ and $B$ as

$$
\begin{aligned}
& \mathcal{M}^{++}=2\left[m_{N} A+\left(E_{N} \sqrt{s}-m_{N}^{2}\right) B\right] \cos (\theta / 2), \\
& \mathcal{M}^{+-}=2\left[E_{N} A+m_{N}\left(\sqrt{s}-E_{N}\right) B\right] \sin (\theta / 2),
\end{aligned}
$$

where $E_{N}$ refers to the energy of the nucleon in the $s$ channel CM system, and $\theta$ is the $s$-channel scattering angle.

We parameterize the invariant amplitudes $A$ and $B$ [27] by

$$
\begin{aligned}
& A=\sum_{i} \beta_{i}^{A}(u) \frac{\zeta_{i}(u)}{\Gamma\left(\alpha_{i}-1 / 2\right)}\left(\frac{s}{s_{0}}\right)^{\alpha_{i}-1 / 2}, \\
& B=\sum_{i} \beta_{i}^{B}(u) \frac{\zeta_{i}(u)}{\Gamma\left(\alpha_{i}-1 / 2\right)}\left(\frac{s}{s_{0}}\right)^{\alpha_{i}-1 / 2},
\end{aligned}
$$

where $s_{0}=1 \mathrm{GeV}^{2}$ is a scaling factor and $\zeta_{i}(u)$ is the Regge propagator,

$$
\zeta_{i}(u)=\frac{1+\mathcal{S}_{i} \exp \left[-i \pi\left(\alpha_{i}(u)-1 / 2\right)\right]}{\sin \left[\pi\left(\alpha_{i}(u)-1 / 2\right)\right]},
$$

with $\mathcal{S}_{i}$ denoting the signature of the trajectory. The $i$-th baryon Regge trajectory, $\alpha_{i}$, is parameterized as a linear function of $u$,

$$
\alpha_{i}(u)=\alpha_{0 i}+\alpha \prime u
$$

where $i$ labels the trajectories $N_{\alpha}, N_{\gamma}, \Delta_{\delta}$ and $\Delta_{\beta}$, respectively. The slope $\alpha$ ' and the intercept $\alpha_{0}$ are determined
Table 1. The leading baryon trajectories. The last column shows the parity partners that have the same signature but opposite parity.

\begin{tabular}{|c|c|c|c|c|}
\hline Trajectory & Isospin & Parity & Signature & Partner \\
\hline$N_{\alpha}$ & $1 / 2$ & + & + & $N_{\beta}$ \\
$N_{\gamma}$ & $1 / 2$ & - & - & $N_{\delta}$ \\
$\Delta_{\delta}$ & $3 / 2$ & + & - & $\Delta_{\gamma}$ \\
$\Delta_{\beta}$ & $3 / 2$ & - & + & $\Delta_{\alpha}$ \\
\hline
\end{tabular}

by a fit to the data. As will be discussed later, we take the same slope parameter for all four trajectories.

The baryon trajectories used in the present work are given in table 1. The signature of a trajectory is defined as $\mathcal{S}=(-1)^{J-1 / 2}$, where $J$ is the baryon spin. The classification of the Regge trajectories is given in terms of the signature $\mathcal{S}= \pm 1$ and the parity $P= \pm 1$. Thus one should consider four trajectories for the nucleon as well as for the Delta-isobar states. Furthermore, the parity partners of the discussed trajectories are defined [35, 36] in the Regge formalism as trajectories with the same signature but opposite parity. They are also indicated in table1. The residue functions $\beta^{A}(u)$ and $\beta^{B}(u)$ for each trajectory are parameterized by

$$
\begin{aligned}
& \beta^{A}(u)=a+b u \\
& \beta^{B}(u)=c+d u
\end{aligned}
$$

\section{Results for high energies}

We use almost all data available for the differential cross sections and polarization asymmetries for backward $\pi N$ scattering with invariant collision energy $\sqrt{s} \geq 3 \mathrm{GeV}$, see tables 6.9] in the appendix for a short overview. For the $\pi^{-} p \rightarrow \pi^{0} n$ reaction (the charge-exchange channel, abbreviated as CEX), the data by Boright et al. 24] and Schneider et al. 37] are known to be inconsistent with the experimental results from DeMarzo et al. 22 and Chase et al. 38. The Regge model analyses [3, 17 done previously included the data from refs. [24,37]. However, in our study we include the experimental results from refs. 22, 38 since these data are more recent and furthermore for these data the appropriate radiative corrections have been applied 22, 27. For $\pi^{+} p \rightarrow \pi^{+} p$ and $\pi^{-} p \rightarrow \pi^{-} p$ backward scattering, the data from refs. $39,40,41,42,43,44$ are not included in our fitting since they are not consistent with the much more recent data indicated in the tables.

The problem of discrepancies in the absolute normalization of the differential cross sections measured in different experiments is discussed in details in refs. [3, 17. In the present analysis we apply the procedure proposed in refs. [3, 17] in order to account for the systematic uncertainties due to the absolute normalization.

Note that in table 7 we indicate references to the data available at invariant collision energies from 2.35 to 3.49 $\mathrm{GeV}$. However, only experimental polarization data at energies above $\sqrt{s}=3 \mathrm{GeV}$ were used in the global fit. 
The other data at low energies are compared with the Regge calculation in order to clarify how much they deviate from the expectation based on the reaction amplitudes constructed at high energies.

Finally, the number of data points (ND) included in the global fit and the obtained $\chi^{2}$ for various observables are listed in table 2. Here we also indicate the $\chi^{2} / \mathrm{ND}$ for different reaction channels. The small $\chi^{2}$ obtained for the fit to the polarization data originates from large uncertainties in the experimental results. By fitting 505 data points we get a total $\chi^{2}=1.84$ per data point.

The 21 free parameters of the model are listed in tab. 3 . Note that the slope $\alpha^{\prime}$ was taken to be the same for the different baryon trajectories. That ensures that the baryon trajectories are parallel in the plane given by the spin and mass of baryons.

Figs. 1] 3] show experimental results on $\pi^{+} p \rightarrow \pi^{+} p$ differential cross sections together with our calculations. Note that the data indicate a dip near $u \simeq-0.15 \mathrm{GeV}^{2}$ where the $N_{\alpha}$ amplitude passes through zero. Indeed taking into account the parameters listed in table 3 it is clear that at $u \simeq-0.15 \mathrm{GeV}^{2}$ the $N_{\alpha}$ trajectory becomes $\alpha(u) \simeq-1 / 2$ and, therefore, the signature factor given by eq. (10) is $\zeta_{\alpha}(u)=0$. However, the dip in the $\pi^{+} p \rightarrow \pi^{+} p$ differential cross sections is partially filled due to the contributions from other trajectories that have zeros in the signature factors at different values of the fourmomentum transfer squared $u$. Also note that at small values of $|u|<0.1 \mathrm{GeV}^{2}$ the data indicate an exponen-

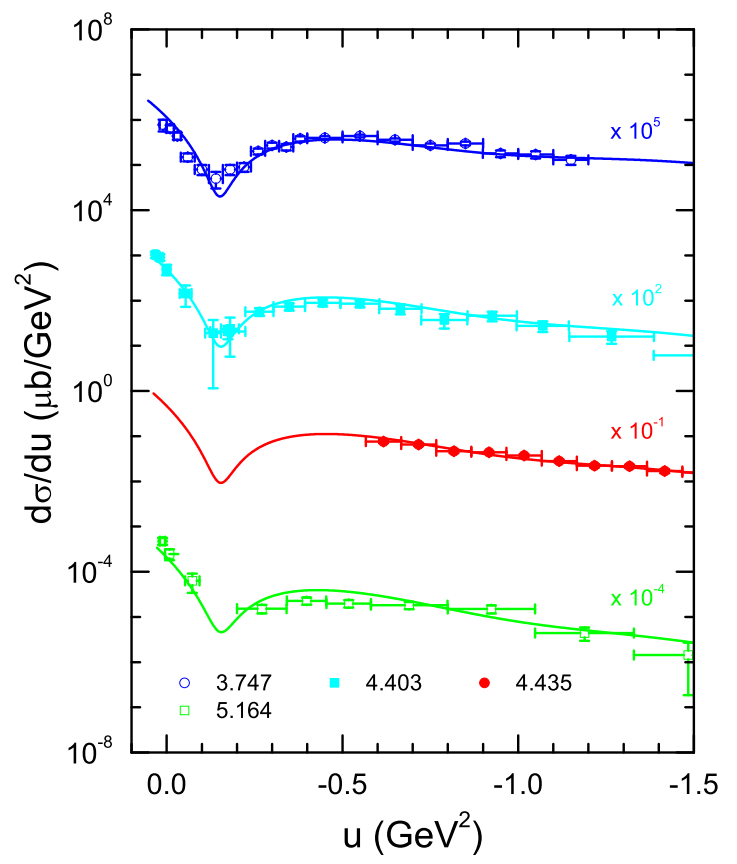

Fig. 1. The differential cross section for $\pi^{+} p \rightarrow \pi^{+} p$ backward scattering as a function of the $u$-channel four-momentum transfer squared shown for different invariant collision energies, $\sqrt{s}$, indicated in the legend. The references to the data are given in tab. 4 The solid lines are the results of our model calculation. Both data and calculations were scaled by the indicated factors.
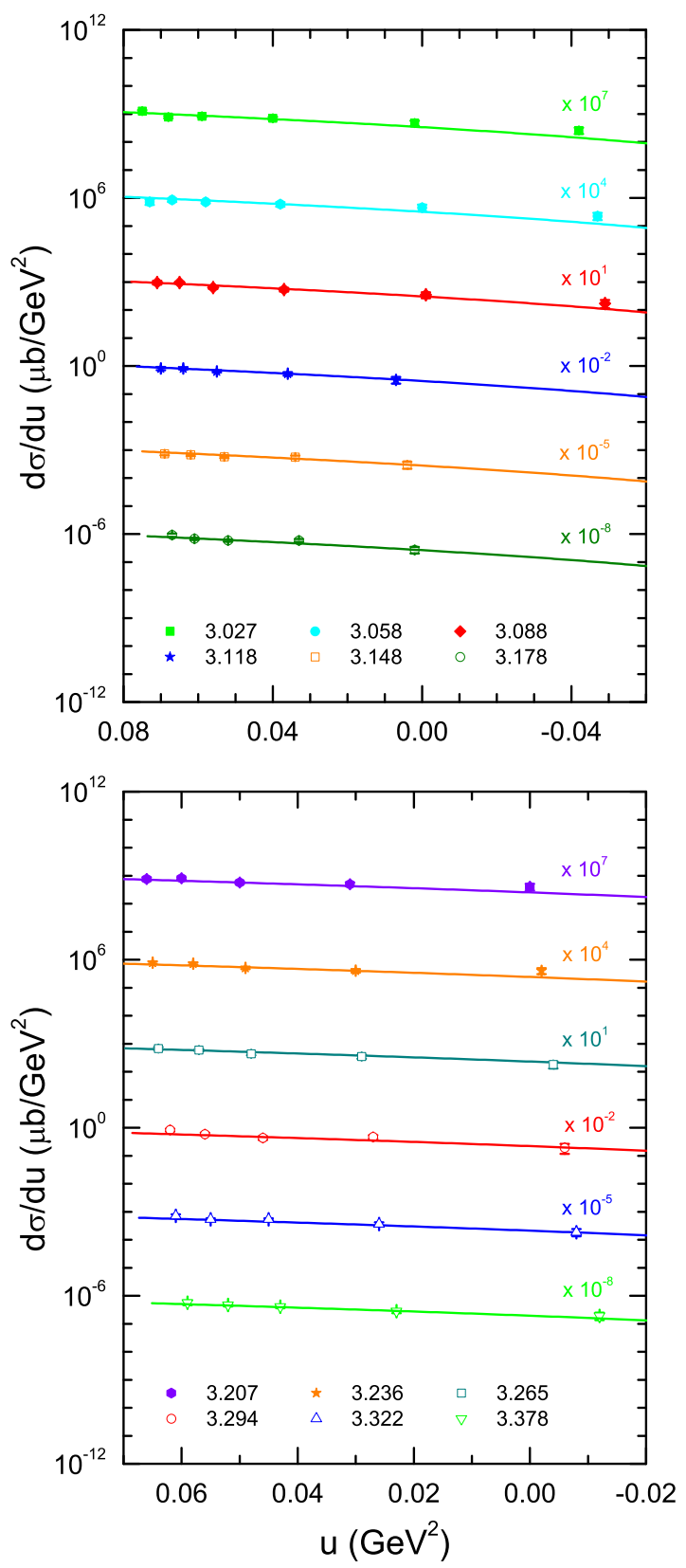

Fig. 2. The differential cross section for $\pi^{+} p \rightarrow \pi^{+} p$ backward scattering as a function of the $u$-channel four-momentum transfer squared shown for different invariant collision energies, $\sqrt{s}$, indicated in the legend. The references to the data are given in tab. 4. The solid lines are the results of our model calculation. Both data and calculations were scaled by the indicated factors.

tial dependence. At $|u|>0.4 \mathrm{GeV}^{2}$ the differential cross sections show a smooth, almost constant behavior. The dip observed in the $\pi^{+} p \rightarrow \pi^{+} p$ differential cross sections allows to conclude that the $N_{\alpha}$-trajectory dominates this reaction channel.

In general there is reasonable agreement between the $\pi^{+} p$ backward scattering data and our Regge calculation. There is, however, a disagreement between our results and 

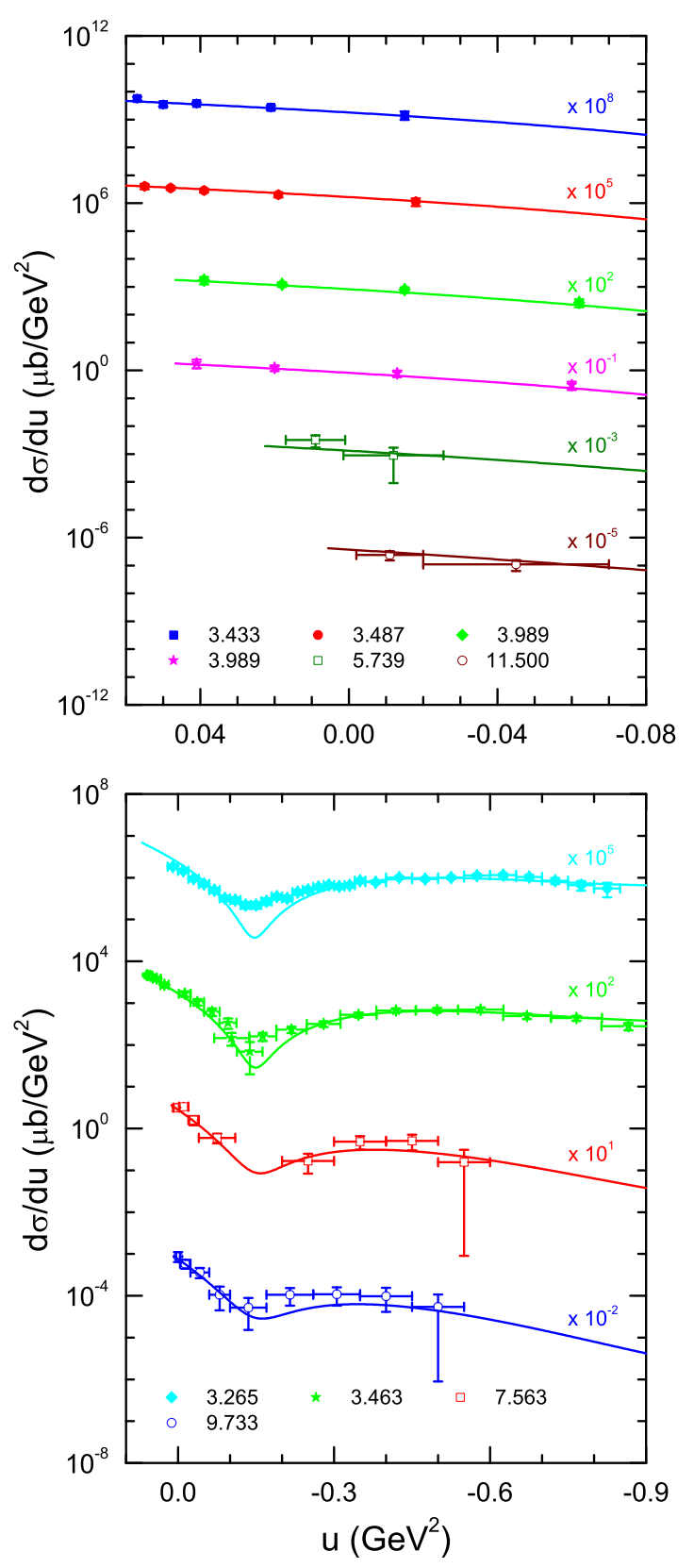

Fig. 3. The differential cross section for $\pi^{+} p \rightarrow \pi^{+} p$ backward scattering as a function of the $u$-channel four-momentum transfer squared shown for different invariant collision energies indicated in the legend. The references to the data are given in tab. 4 The solid lines show the results of our model calculation. Both data and calculations were scaled by the indicated factors.

the data of ref. 45] at $\sqrt{s}=3.265 \mathrm{GeV}$ in the vicinity of the dip, which signals that the Regge approximation starts to break down for low energies.

Figs.44 5 illustrate our calculation together with experimental results on $\pi^{-} p \rightarrow \pi^{-} p$ differential cross sections. Now the data do not have a dip structure but rather show a smooth $u$-dependence. There is no $N_{\alpha}$-trajectory con-

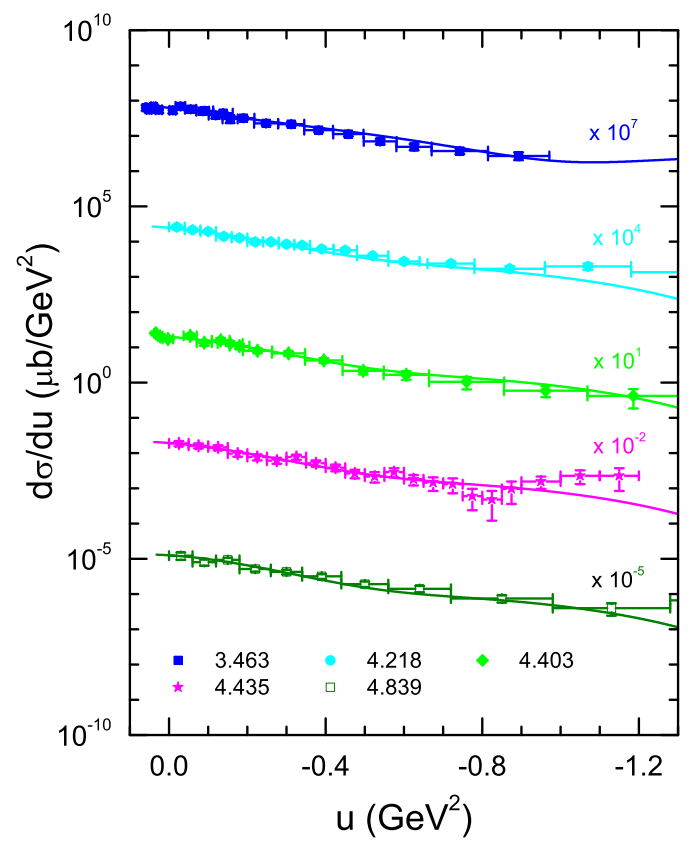

Fig. 4. The differential cross section for $\pi^{-} p \rightarrow \pi^{-} p$ backward scattering as a function of the $u$-channel four-momentum transfer squared shown for different invariant collision energies indicated in the legend. The references to the data are given in tab. 6. The solid lines are the results of our model calculation. Both data and calculations were scaled by the indicated factors.

tribution to this reaction channel. The reaction is entirely governed by the $\Delta$-trajectories.

Taking the parameters from table 3 one might expect that in the scattering region the first zero of the $\Delta_{\delta}$ trajectory is located around $u \simeq-1.68 \mathrm{GeV}^{2}$, while the first zero of the $\Delta_{\beta}$-trajectory is around $u \simeq-2 \mathrm{GeV}^{2}$. There are no data available at these four-momentum transfers to clarify the situation. And, moreover, at these large values of $|u|$ one should expect additional contributions from the $t$ and $s$ channel exchanges too.

It is interesting that the data shown in fig. 4 indicate some increase of the differential cross section at $|u|>0.8$ $\mathrm{GeV}^{2}$, although the accuracy of the experimental results is not so high. The model calculation does not produce

Table 2. Summary of the $\chi^{2}$ for the differential cross sections and polarization data for $\pi N$ backward scattering. Here ND denotes the number of data points.

\begin{tabular}{|l|r|c|}
\hline Observable & ND & $\chi^{2} / \mathrm{ND}$ \\
\hline$d \sigma / d u\left(\pi^{+} p\right)$ & 227 & 2.32 \\
$d \sigma / d u\left(\pi^{-} p\right)$ & 187 & 1.43 \\
$d \sigma / d u(\mathrm{CEX})$ & 59 & 1.94 \\
$P\left(\pi^{+} p\right)$ & 20 & 0.71 \\
$P\left(\pi^{-} p\right)$ & 12 & 0.65 \\
\hline Total & 505 & 1.84 \\
\hline
\end{tabular}



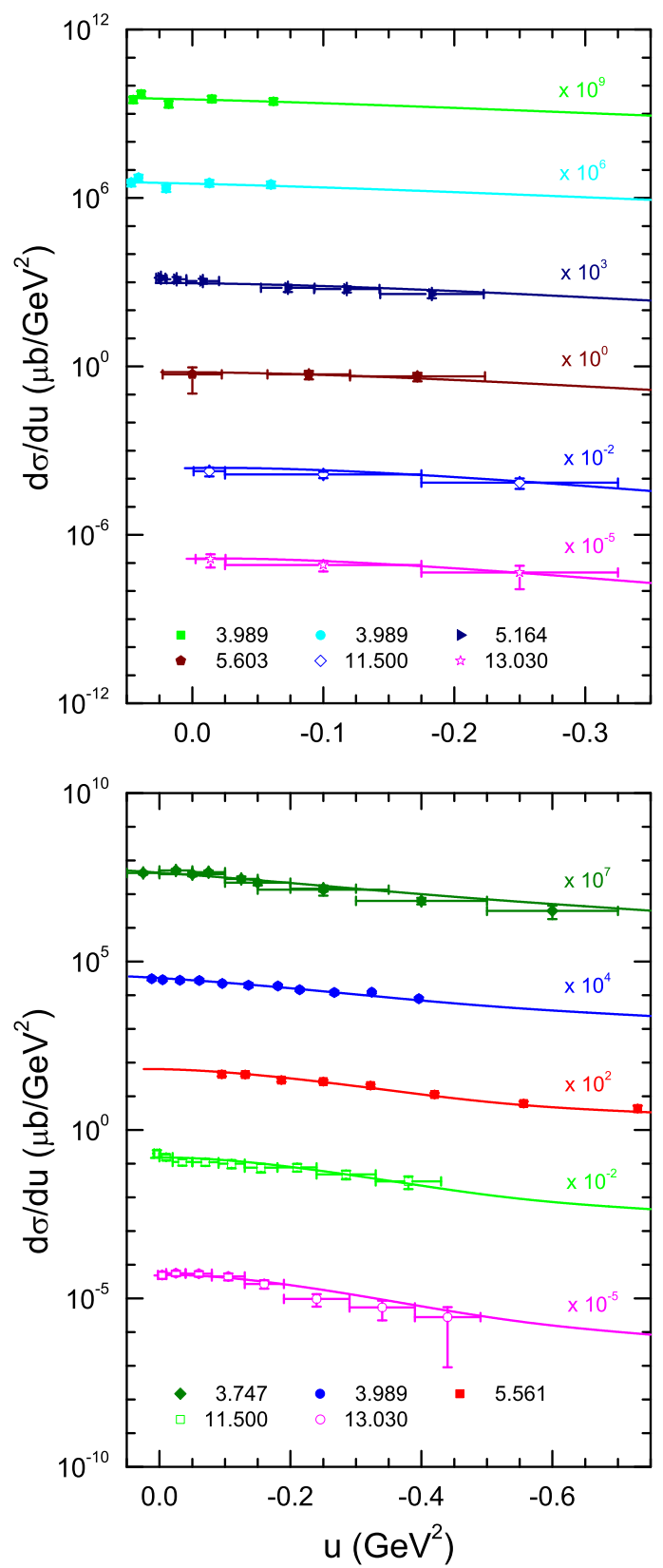

Fig. 5. The differential cross section for $\pi^{-} p \rightarrow \pi^{-} p$ backward scattering as a function of the $u$-channel four-momentum transfer squared shown for different invariant collision energies indicated in the legend. The references to the data are given in tab. 6] The solid lines are the results of our model calculation. Both data and calculations were scaled by the indicated factors.

such a trend but rather suggests that the differential cross sections slightly decrease.

The differential cross section for the $\pi^{-} p \rightarrow \pi^{0} n$ charge exchange reaction is shown in fig. 6. The data indicate a dip around $u \simeq-0.15 \mathrm{GeV}$ which originates from the $N_{\alpha}$-trajectory. However, the structure of the dip observed in the charge exchange reaction differs from the one seen in the $\pi^{+} p \rightarrow \pi^{+} p$ differential cross section. Historically this difference motivated the inclusion of an additional

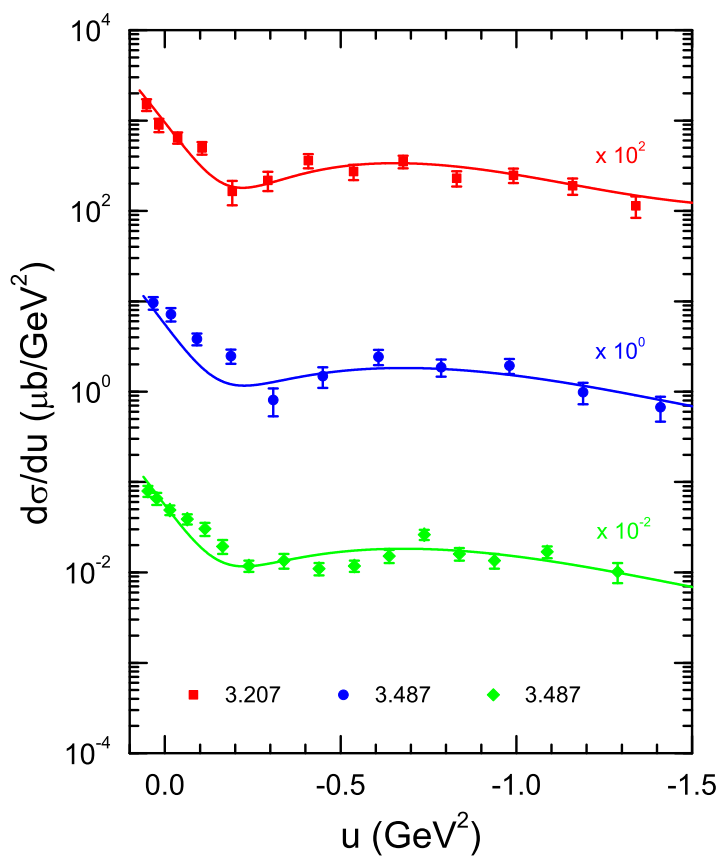

Fig. 6. The differential cross section for $\pi^{-} p \rightarrow \pi^{0} n$ backward scattering as a function of the $u$-channel four-momentum transfer squared shown for different invariant collision energies indicated in the legend. The references to the data are given in tab. 5. The solid lines are the results of our model calculation. Both data and calculations were scaled by the indicated factors.

Table 3. Parameters of the $N_{\alpha}, N_{\gamma}, \Delta_{\delta}$ and $\Delta_{\beta}$ amplitudes obtained in the global fit. Note that the slope $\alpha^{\prime}$ was taken to be the same for the different amplitudes.

\begin{tabular}{|r|r|r|r|r|}
\hline Parameters & $N_{\alpha}$ & $N_{\gamma}$ & $\Delta_{\delta}$ & $\Delta_{\beta}$ \\
\hline$a\left[\mathrm{GeV}^{-1}\right]$ & -60.68 & 47.22 & -75.15 & 1419.99 \\
$b\left[\mathrm{GeV}^{-3}\right]$ & 326.52 & -215.84 & -138.75 & 3052.84 \\
$c\left[\mathrm{GeV}^{-2}\right]$ & 546.40 & -101.11 & 64.16 & -192.64 \\
$d\left[\mathrm{GeV}^{-4}\right]$ & 307.42 & -128.04 & 86.77 & -695.81 \\
$\alpha_{0}$ & -0.36 & -0.62 & 0.03 & -2.65 \\
\hline$\alpha^{\prime}\left[\mathrm{GeV}^{-2}\right]$ & \multicolumn{5}{|c|}{0.908} \\
\hline
\end{tabular}

$N_{\gamma}$-trajectory in the Regge analysis of pion-nucleon backward scattering. Our Regge model describes the differential cross sections available for the $\pi^{-} p \rightarrow \pi^{0} n$ reaction fairly well.

Concerning the differential cross sections we find that our Regge calculation reproduces the data available for $\pi^{+} p \rightarrow \pi^{+} p, \pi^{-} p \rightarrow \pi^{-} p$ and $\pi^{-} p \rightarrow \pi^{0} n$ backward scattering reasonably well and thus corroborates the finding of the previous analyses [1,2,3,4,5, that, in principle, three trajectories, namely $N_{\alpha}, N_{\gamma}$ and $\Delta_{\delta}$ play a significant role in describing the data on differential cross sections. The most striking feature of the data is the dip observed for the $\pi^{+} p \rightarrow \pi^{+} p$ and $\pi^{-} p \rightarrow \pi^{0} n$ reaction. This dip allows to determine the intercept $\alpha_{0}$ of the $N_{\alpha}$-trajectory. At the same time there is no dip in the $\pi^{-} p \rightarrow \pi^{-} p$ back- 


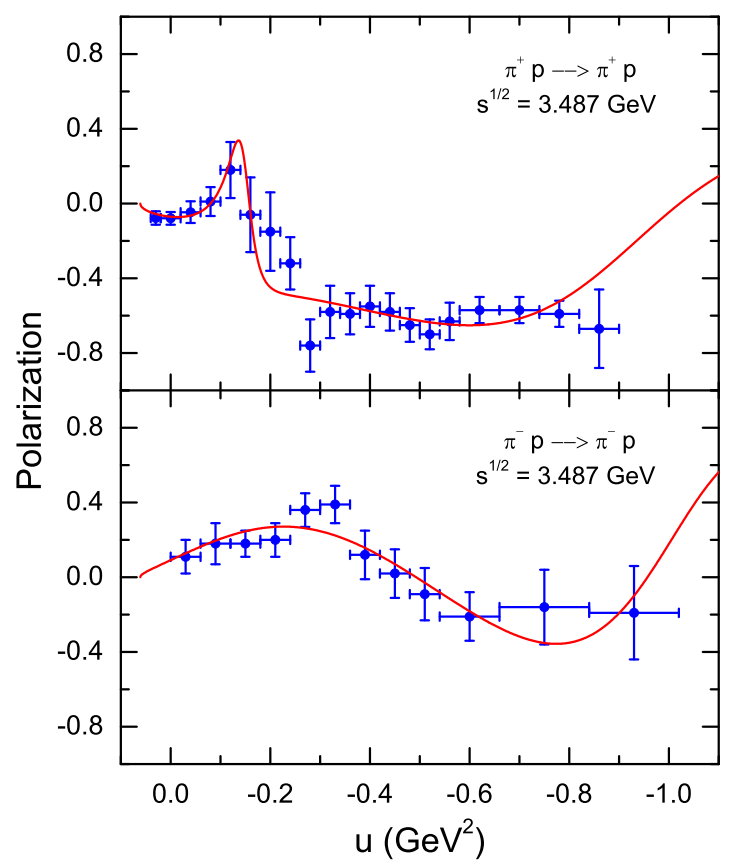

Fig. 7. The polarization for $\pi^{+} p \rightarrow \pi^{+} p$ and $\pi^{-} p \rightarrow$ $\pi^{-} p$ backward scattering at the pion beam momentum of 6 $\mathrm{GeV}(\sqrt{s}=3.49 \mathrm{GeV})$ as a function of the $u$-channel fourmomentum transfer squared. The references to the data are specified in table 7. The solid lines are the results of our model calculation.

ward scattering since there is no contribution from the $N_{\alpha}$-trajectory in that reaction channel.

Fig. 7 shows the polarization for $\pi^{+} p \rightarrow \pi^{+} p$ and $\pi^{-} p \rightarrow \pi^{-} p$ backward scattering. These data were collected 13, 14] at the pion beam momentum of $6 \mathrm{GeV}$ corresponding to an invariant collision energy of $\sqrt{s}=3.49 \mathrm{GeV}$. For both reactions the polarization substantially depends on the four-momentum transfer squared $u$. Note that only the $\Delta$-trajectories contribute to the $\pi^{-} p \rightarrow \pi^{-} p$ backward scattering. Therefore, within models that only take into account the $\Delta_{\delta}$-trajectory, it is impossible to reproduce the polarization for the $\pi^{-} p \rightarrow \pi^{-} p$ reaction. Previous analyses [1, 2, 3, 4, 5] made many ad hoc assumptions, but did not manage to achieve any consistent fits. The present work includes the $\Delta_{\beta}$-trajectory, which allows to reproduce the polarization data.

\section{Extrapolation below $3 \mathrm{GeV}$}

Fig. 8 shows the energy dependence of the differential cross section for the reactions $\pi^{+} p \rightarrow \pi^{+} p, \pi^{-} p \rightarrow \pi^{-} p$ and $\pi^{-} p \rightarrow \pi^{0} p$ measured at the pion scattering angle of $180^{\circ}$. The data indicate considerable structures for center-ofmass energies up to $\simeq 3 \mathrm{GeV}$ or even higher. There were intense discussions 227,46, 47, 48 whether these structures originate from the excitation of high mass baryons. Indeed, these data on pion scattering at $180^{\circ}$ seem to be up to now the only direct evidence of the existence of excited baryons with masses above $2.4 \mathrm{GeV}$.

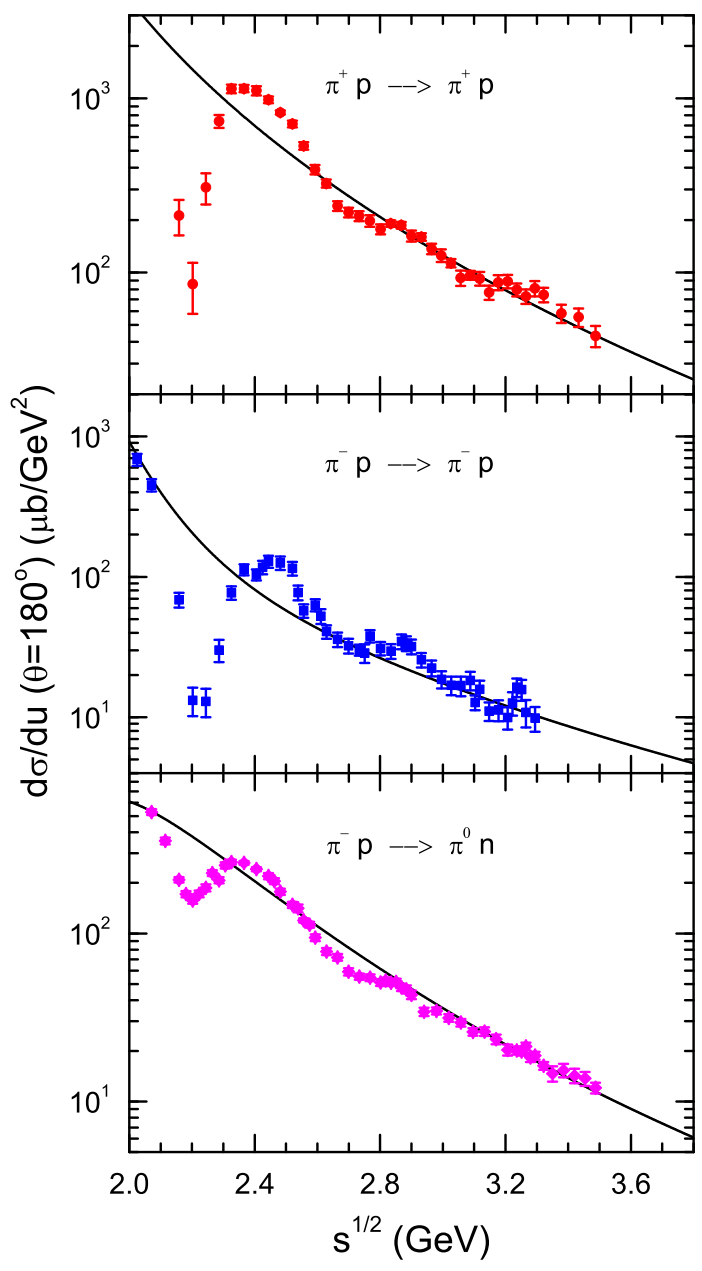

Fig. 8. Differential cross section for $\pi^{+} p \rightarrow \pi^{+} p, \pi^{-} p \rightarrow \pi^{-} p$ and $\pi^{-} p \rightarrow \pi^{0} n$ reaction at the scattering angle $\theta=180^{\circ}$ as a function of the invariant collision energy. The references to the data are shown in table 8 . The solid lines are the results of our model calculation.

The lines in fig. 8 show the results of our calculation extrapolated to low energies. Note that the data below $3 \mathrm{GeV}$ were not included in our fit. We observe that the data oscillate around the non-resonant continuum given by the Regge amplitude. Above $\simeq 3 \mathrm{GeV}$ our calculations approach the experimental data. Although the differential cross section of the $\pi^{-} p \rightarrow \pi^{-} p$ reaction indicates some fluctuations above $\simeq 3 \mathrm{GeV}$ we consider this as being due to statistical uncertainties. This point will be illustrated below. Fig. 9 displays the data available for the $\pi^{+} p \rightarrow$ $\pi^{+} p, \pi^{-} p \rightarrow \pi^{-} p$ and $\pi^{-} p \rightarrow \pi^{0} n$ reactions at the fourmomentum transfer squared $u=0 \mathrm{GeV}^{2}$ as a function of energy. One sees that the Regge approach agrees with the data above $\sqrt{s} \simeq 3 \mathrm{GeV}$.

Next we investigate whether the fluctuations of the data with respect to our Regge calculation shown in fig. 8 for the energies $2 \leq \sqrt{s} \leq 3.5 \mathrm{GeV}$ is of systematic or of statistical nature. For that purpose, we evaluate the difference $D$ between the experimental differential cross sections and those predicted by the Regge model at the 


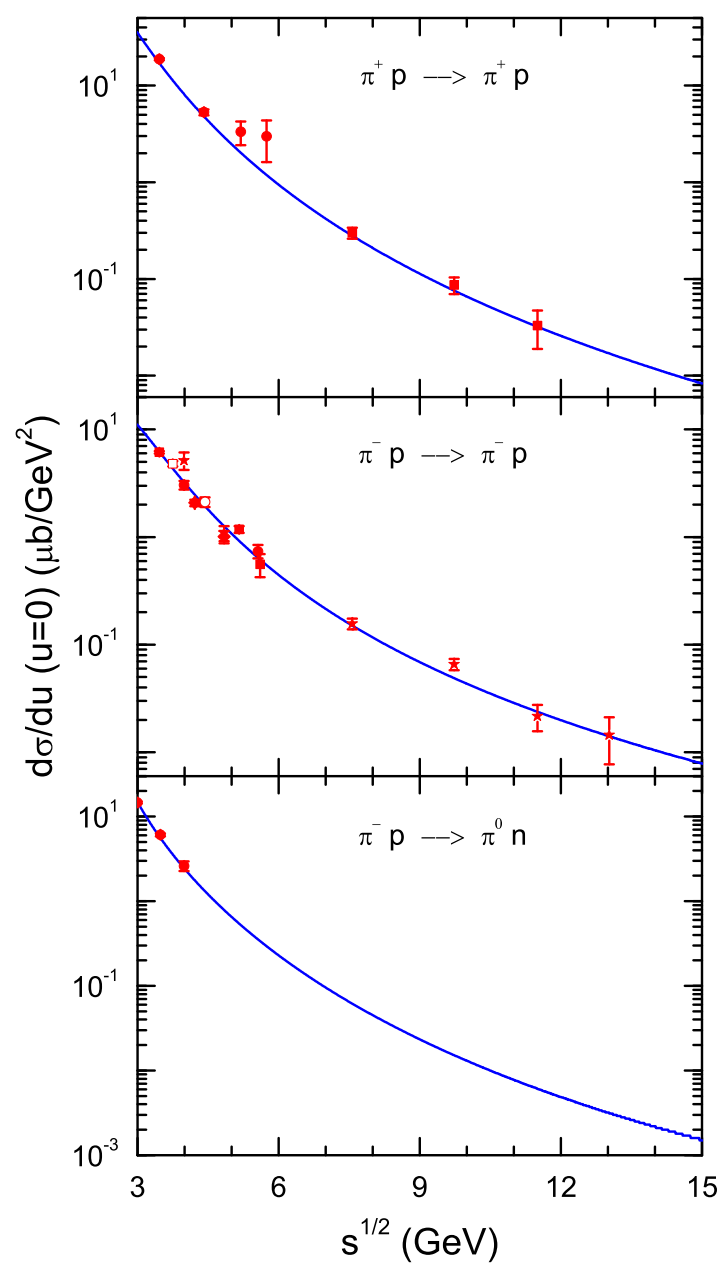

Fig. 9. Differential cross section for $\pi^{+} p \rightarrow \pi^{+} p, \pi^{-} p \rightarrow \pi^{-} p$ and $\pi^{-} p \rightarrow \pi^{0} n$ scattering at the four-momentum transfer squared $u=0$ as a function of the invariant collision energy. The references to the data are shown in table 9 The solid lines show our results.

scattering angle $\theta=180^{\circ}$ at each $\sqrt{s}$ and for each reaction channel, i. e.

$$
D=\frac{d \sigma^{\text {Exp. }}}{d u}-\frac{d \sigma^{\text {Regge }}}{d u}
$$

and present the results in fig. 10 using a linear scale.

At energies $\sqrt{s}>2.8 \mathrm{GeV}$ the data available for $\pi^{+} p \rightarrow$ $\pi^{+} p, \pi^{-} p \rightarrow \pi^{-} p$ and $\pi^{-} p \rightarrow \pi^{0} n$ scattering at $\theta=180^{0}$ are consistent with the predictions of our Regge calculation. Below that energy the data indicate some room for additional contributions. At least the magnitude of the variations seem to be larger than the statistical fluctuations of the experimental results. Furthermore, the different $\pi N$ reaction channels do not indicate the same pattern of differences between the data and the Regge results. The largest difference occurs for the reaction $\pi^{+} p \rightarrow \pi^{+} p$ at $180^{\circ}$, while the values of $D$ obtained for $\pi^{-} p \rightarrow \pi^{-} p$ and $\pi^{-} p \rightarrow \pi^{0} n$ are almost identical. Note that in the $s$-channel only excitations of $\Delta$-isobars is possible for the $\pi^{+} p \rightarrow \pi^{+} p$ reaction. The two other reactions allow for

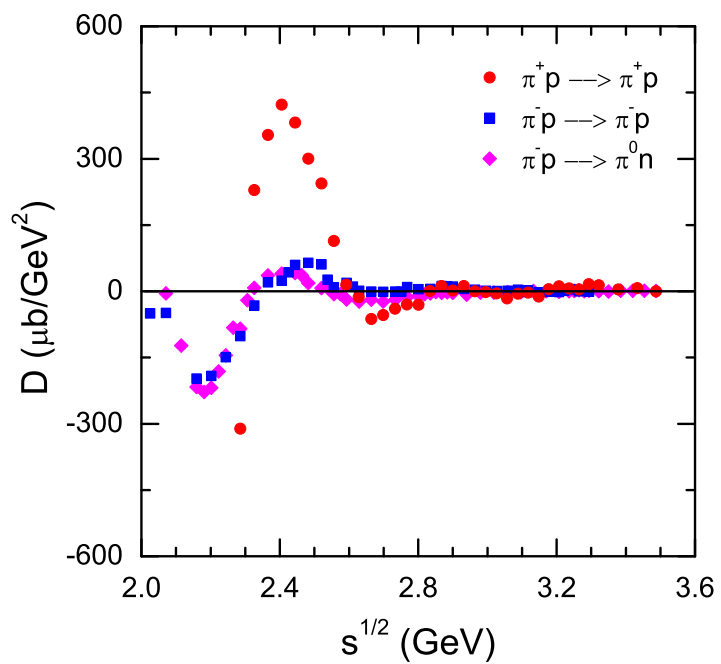

Fig. 10. The difference $D$ between the experimental differential cross sections and the Regge calculation at the scattering angle $\theta=180^{\circ}$ as defined by eq. (14), shown as a function of the invariant collision energy for different reaction channels.

the excitation of nucleon as well as $\Delta$-resonances in the $s$-channel.

It is interesting to illustrate the arguments of ref. 46 where similar differences between the Regge predictions and data for $\pi^{+} p$ elastic scattering at $180^{\circ}$ were evaluated in terms of an additional resonance contribution. Note that at $180^{\circ}$ only the spin non-flip amplitude contributes, see eq. (77). The amplitude due to the excitation of baryon resonances in the $s$-channel is given by

$$
\mathcal{M}^{++}=\sum_{n} \frac{C_{n} X_{n}(J+1 / 2)}{\epsilon-i}(-1)^{l},
$$

where the summation is done over the resonances, $C_{n}$ is a Clebsch-Gordan coefficient, $X_{n}$ denotes the resonance elasticity, $J$ the spin of the resonance, and $l$ the orbital momentum between pion and nucleon. Here

$$
\epsilon=\frac{M_{R}^{2}-s}{M_{R} \Gamma_{R}}
$$

with $M_{R}$ and $\Gamma_{R}$ being the resonance mass and width, respectively. The width was taken as energy-dependent. Furthermore, recalling $P_{l}(\cos \theta=-1)=(-1)^{l}$, one sees explicitly that the resonance amplitudes interfere with the non-resonant amplitude either constructively or destructively according to the parity of the resonance. In the case of $\pi^{+} p \rightarrow \pi^{+} p$ elastic scattering the $s$-channel contributions from the $D_{35}(2350)$ and $H_{3,11}(2420)$ with $l=2$ and $l=5$ are the major candidates responsible for the change of the sign of $D$ seen in fig. 10.

As is illustrated by fig. 7 the Regge model reproduces the polarization data for $\pi^{+} p \rightarrow \pi^{+} p$ and $\pi^{-} p \rightarrow \pi^{-} p$ backward scattering at $\sqrt{s}=3.49 \mathrm{GeV}$.

In fig. 11, we show the polarization data for backward scattering in the reaction $\pi^{+} p \rightarrow \pi^{+} p$ available in the energy range $2.4<\sqrt{s}<3 \mathrm{GeV}$ [21,49]. For energies 


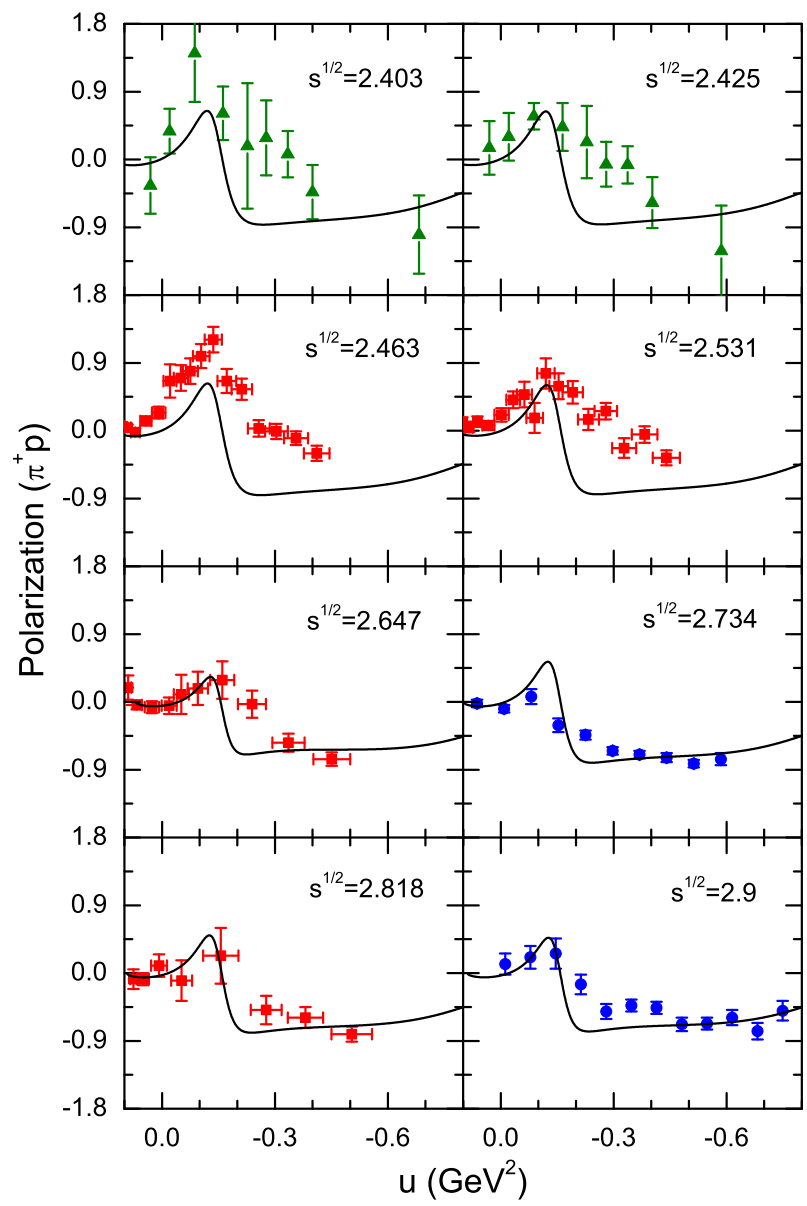

Fig. 11. The polarization asymmetry for $\pi^{+} p \rightarrow \pi^{+} p$ backward scattering at different invariant collision energies as indicated. The references to the data are specified in tab. 7 The solid lines show the results of our Regge calculation with the model parameters listed in table 3

above $\sqrt{s} \simeq 2.73 \mathrm{GeV}$ the Regge results reproduce the polarization data reasonably well, but within the range $2.46 \leq \sqrt{s} \leq 2.64 \mathrm{GeV}$, there is room for additional contributions at $|u|>0.1 \mathrm{GeV}^{2}$. This finding is consistent with the data on differential cross section presented in fig. 8 ,

The situation is quite different for the polarization observed in $\pi^{-} p \rightarrow \pi^{-} p$ backward scattering [50,15]. The data [50] at $\sqrt{s} \simeq 2.45 \mathrm{GeV}$ show a $u$-dependence similar to the one given by the Regge model, but with a systematic shift to negative values. The data at $\sqrt{s}=2.65 \mathrm{GeV}$ and $\sqrt{s}=2.73 \mathrm{GeV}$ indicate an almost zero polarization within the experimental uncertainties. This is in line with the $\pi^{-} p \rightarrow \pi^{-} p$ data on the differential cross section at $180^{\circ}$ displayed in fig. 8 .

Let us finally come to the baryon trajectories. Fig. 13 shows the Chew-Frautschi plot for the $N_{\alpha}$ trajectory. The poles of the amplitude of eq. (9) correspond to baryons with spin $J$, as is indicated by the dashed lines. The results of partial wave analyses (PWA) from the KarlsruheHelsinki (KH) 27,30 and the George Washington University (GWU) 51] groups are included in the figure, too.

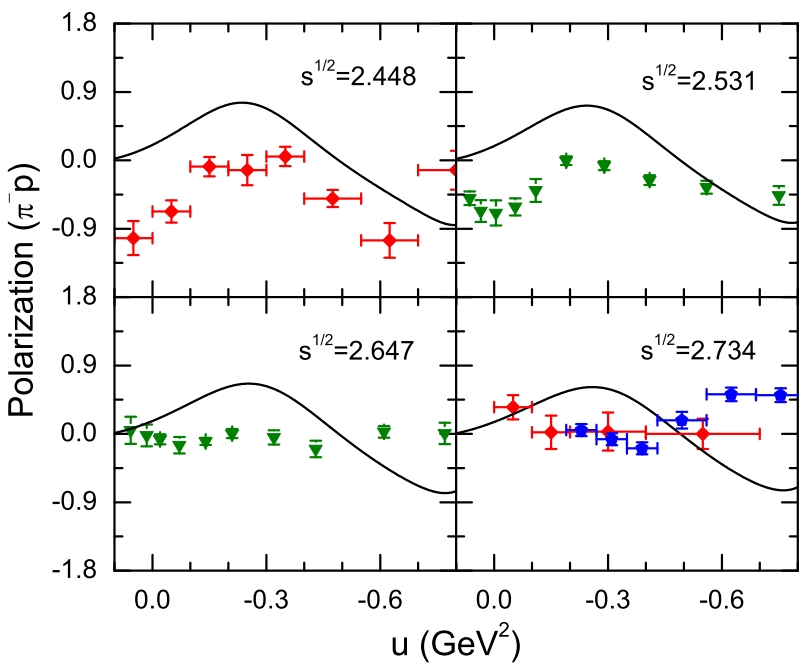

Fig. 12. The polarization asymmetry for $\pi^{-} p \rightarrow \pi^{-} p$ backward scattering at different invariant collision energies as indicated. The references to the data are specified in table 7 The solid lines show the results of our Regge calculation with the model parameters listed in table 3 .

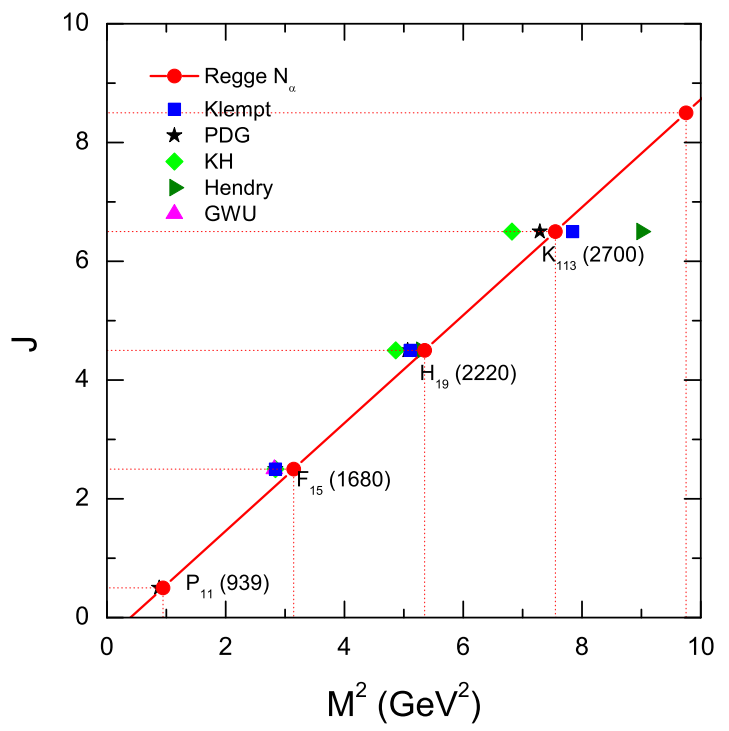

Fig. 13. Chew-Frautschi plot for the $N_{\alpha}$ trajectory for baryons with parity $P=+1$ and signature $\mathcal{S}=+1$. The line shows the Regge trajectory according to table 3 . The symbols indicate the results from other approaches.

We also indicate the averaged values given by the Particle Data Group [52] and the most recent systematic analysis by Klempt and Richard [53. The analysis by Hendry [28, 48] is based on an impact parameter approach that differs significantly from our result for the $K_{1,13}(2700)$.

Fig. 14] shows the Chew-Frautschi plot for the $N_{\gamma}$ trajectory. There are two resonances with a four-star rating by the PDG on this trajectory, and even the $I_{1,11}(2600)$ is classified with three stars.

The $\Delta_{\delta}$ and $\Delta_{\beta}$-trajectories are shown in figs. [15] and 16. respectively. The mass of the $H_{3,11}(2420)$ isobar obtained by the GWU PWA differs significantly from other 


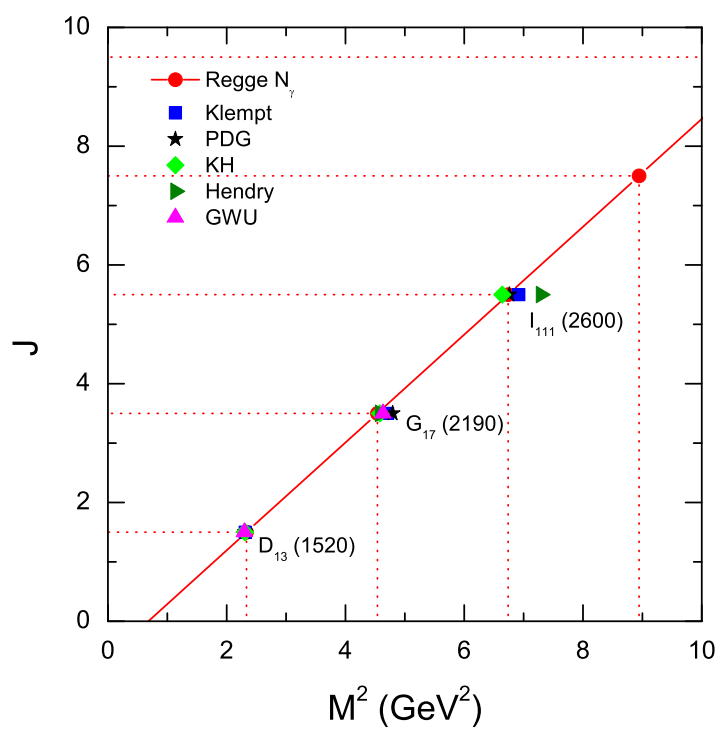

Fig. 14. $N_{\gamma}$ trajectory for baryons with parity $P=-1$ and signature $\mathcal{S}=-1$. The line shows the Regge trajectory according to table 3 The symbols indicate the results from other approaches.

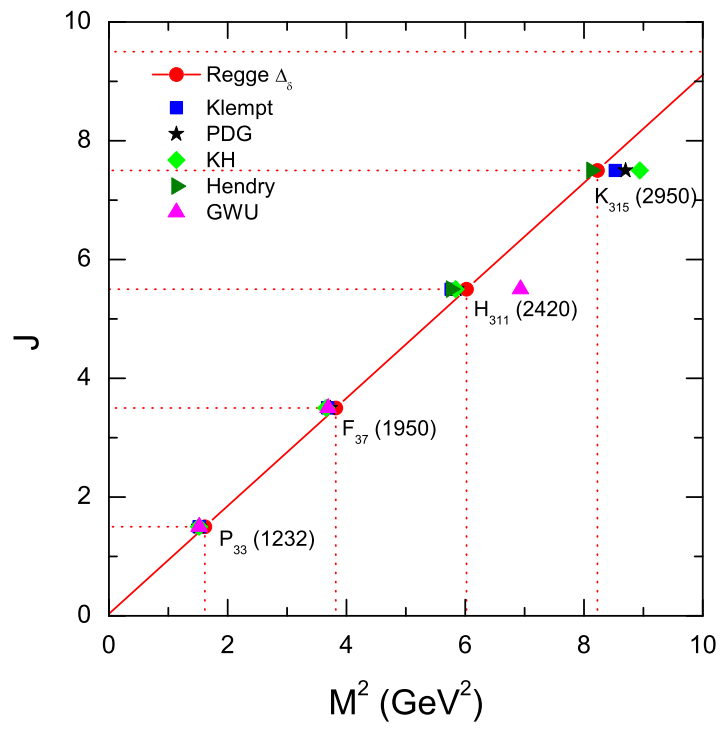

Fig. 15. $\Delta_{\delta}$ trajectory for $\Delta$-isobars with parity $P=+1$ and signature $\mathcal{S}=-1$. The line shows the Regge trajectory, while the symbols indicate the results from other approaches.

results. Our analysis suggests a $G_{39}$ resonance with a mass of $2.83 \mathrm{GeV}$ as member of the $\Delta_{\beta}$ trajectory.

\section{Summary}

We have performed a systematic analysis of the data on differential cross sections and polarizations available for $\pi^{+} p \rightarrow \pi^{+} p, \pi^{-} p \rightarrow \pi^{-} p$ and $\pi^{-} p \rightarrow \pi^{0} n$ scattering at backward angles. We started out from a Regge model including the $N_{\alpha}, N_{\gamma}, \Delta_{\delta}$ and $\Delta_{\beta}$ trajectories and determined the reaction amplitude at energies $\sqrt{s}>3 \mathrm{GeV}$.

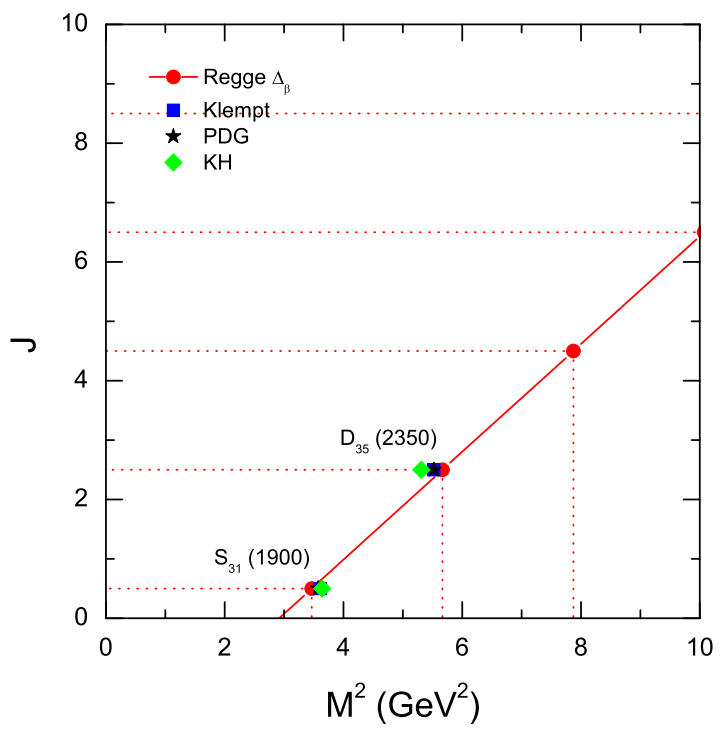

Fig. 16. $\Delta_{\beta}$ trajectory for $\Delta$-isobars with parity $P=-1$ and signature $\mathcal{S}=+1$. The line shows the Regge trajectory, while the symbols indicate the results from other approaches.

In contrast to previous analyses, the present study reproduces the polarization asymmetriess for both $\pi^{+} p$ and $\pi^{-} p$ backward elastic scattering within standard Regge phenomenology. We found that it is not necessary to resort to non-Regge terms 4, 16, 17,5, 18 to describe the polarization, but rather that it is important to include the $\Delta_{\beta}$ trajectory which was neglected in previous analyses.

After the reaction amplitude was fixed at high energies we have inspected the data on differential cross sections for scattering at $\theta=180^{\circ}$ and the polarization asymmetry at energies $2 \leq \sqrt{s} \leq 3 \mathrm{GeV}$. The data available at $\theta=180^{\circ}$ are of special interest because the $\pi^{+} p \rightarrow \pi^{+} p$, $\pi^{-} p \rightarrow \pi^{-} p$, and $\pi^{-} p \rightarrow \pi^{0} n$ differential cross sections indicate considerable structures for center-of-mass energies up to $\simeq 3 \mathrm{GeV}$. The data fluctuate around the cross sections given by the Regge calculation. This can be considered as direct evidence of the excitation of baryons with masses up to approximately $2.8 \mathrm{GeV}$. This point of view is further supported by the data on the polarization asymmetry available at these energies.

This work is partially supported by the Helmholtz Association through funds provided to the virtual institute "Spin and strong QCD" (VH-VI-231), by the EU Integrated Infrastructure Initiative HadronPhysics2 Project (WP4 QCDnet) and by DFG (SFB/TR 16, "Subnuclear Structure of Matter"). This work was also supported in part by U.S. DOE Contract No. DE-AC05-06OR23177, under which Jefferson Science Associates, LLC, operates Jefferson Lab. F.H. is grateful to the support from the Alexander von Humboldt Foundation during his stay in Jülich where the main part of this paper was completed and the support by COSY FFE grant No. 41445282 (COSY-058). A.S. acknowledges support by the JLab grant SURA-06-C0452 and the COSY FFE grant No. 41760632 (COSY-085). 


\section{Appendix: Data collection}

The appendix summarizes the world data on backward pion-nucleon scattering.

Table 4. References to data on $\pi^{+} p \rightarrow \pi^{+} p$ differential cross sections for backward scattering.

\begin{tabular}{|c|c|c|c|c|c|}
\hline $\begin{array}{l}\sqrt{s} \\
\mathrm{GeV}\end{array}$ & $\begin{array}{c}p_{\pi} \\
\mathrm{GeV}\end{array}$ & $\begin{array}{r}u_{\min } \\
\mathrm{GeV}^{2}\end{array}$ & $\begin{array}{r}u_{\max } \\
\mathrm{GeV}^{2}\end{array}$ & Experiment & Ref \\
\hline 3.02 & 4.4 & -0.04 & 0.07 & Lennox 75 & 46 \\
\hline 3.06 & 4.5 & -0.05 & 0.07 & Lennox 75 & 46 \\
\hline 3.09 & 4.6 & -0.05 & 0.07 & Lennox 75 & 46 \\
\hline 3.12 & 4.7 & 0.01 & 0.07 & Lennox 75 & 46 \\
\hline 3.15 & 4.8 & 0.00 & 0.07 & Lennox 75 & 46 \\
\hline 3.18 & 4.9 & 0.00 & 0.07 & Lennox 75 & 46 \\
\hline 3.21 & 5.0 & 0.00 & 0.07 & Lennox 75 & 46 \\
\hline 3.24 & 5.1 & -0.00 & 0.07 & Lennox 75 & 46 \\
\hline 3.26 & 5.2 & -0.00 & 0.06 & Lennox 75 & 46 \\
\hline 3.26 & 5.2 & -0.82 & 0.01 & Baker 71 & 45 \\
\hline 3.29 & 5.3 & -0.01 & 0.06 & Lennox 75 & 46 \\
\hline 3.32 & 5.4 & -0.01 & 0.06 & Lennox 75 & 46 \\
\hline 3.38 & 5.6 & -0.01 & 0.06 & Lennox 75 & 46 \\
\hline 3.43 & 5.8 & -0.01 & 0.06 & Lennox 75 & 46 \\
\hline 3.46 & 5.9 & -0.87 & 0.06 & Owen 69 & 23 \\
\hline 3.49 & 6.0 & -0.02 & 0.06 & Lennox 75 & 46 \\
\hline 3.75 & 7.0 & -1.15 & 0.01 & Baker 71 & 45 \\
\hline 3.99 & 8.0 & -0.06 & 0.04 & Frisken 65 & 54 \\
\hline 3.99 & 8.0 & -0.06 & 0.04 & Orear 66 & 55 \\
\hline 4.40 & 9.8 & -2.29 & 0.03 & Owen 69 & 23 \\
\hline 4.43 & 10.0 & -17.45 & -0.62 & Baglin 75 & 7 \\
\hline 5.16 & 13.7 & -2.82 & 0.01 & Owen 69 & 23 \\
\hline 5.74 & 17.1 & -0.01 & 0.01 & Owen 69 & 23 \\
\hline 7.56 & 30.0 & -0.55 & 0.00 & Baker 83 & 10 \\
\hline 9.73 & 50.0 & -0.50 & 0.00 & Baker 83 & 10 \\
\hline 11.50 & 70.0 & -0.05 & -0.01 & Baker 83 & 10 \\
\hline
\end{tabular}

Table 5. References to data on $\pi^{-} p \rightarrow \pi^{0} n$ differential cross sections for backward scattering.

\begin{tabular}{|c|r|r|r|l|l|}
\hline $\begin{array}{l}\sqrt{s} \\
\mathrm{GeV}\end{array}$ & $\begin{array}{c}p_{\pi} \\
\mathrm{GeV}\end{array}$ & $\begin{array}{c}u_{\min } \\
\mathrm{GeV}^{2}\end{array}$ & $\begin{array}{c}u_{\max } \\
\mathrm{GeV}^{2}\end{array}$ & Experiment & Ref. \\
\hline 3.21 & 5.0 & -1.91 & 0.05 & Chase 70 & {$[38$} \\
3.49 & 6.0 & -2.12 & 0.03 & Chase 70 & {$[38$} \\
3.49 & 6.0 & -1.29 & 0.05 & DeMarzo 75 & 22 \\
\hline
\end{tabular}

Table 6. References to data on $\pi^{-} p \rightarrow \pi^{-} p$ differential cross sections for backward scattering.

\begin{tabular}{|c|c|c|c|c|c|}
\hline $\begin{array}{l}\sqrt{s} \\
\mathrm{GeV}\end{array}$ & $\begin{array}{c}p_{\pi} \\
\mathrm{GeV}\end{array}$ & $\begin{array}{r}u_{\min } \\
\mathrm{GeV}^{2}\end{array}$ & $\begin{array}{r}u_{\max } \\
\mathrm{GeV}^{2}\end{array}$ & Experiment & Ref. \\
\hline 3.46 & 5.9 & -0.89 & 0.06 & Owen 69 & 23 \\
\hline 3.75 & 7.0 & -0.60 & 0.02 & Baker 71 & 45 \\
\hline 3.99 & 8.0 & -0.39 & 0.01 & Anderson 68 & 56 \\
\hline 3.99 & 8.0 & -0.06 & 0.04 & Frisken 65 & 54 \\
\hline 3.99 & 8.0 & -0.06 & 0.05 & Orear 66 & 55 \\
\hline 4.22 & 9.0 & -1.40 & -0.02 & Jacholkowski 77 & 8 \\
\hline 4.40 & 9.8 & -2.39 & 0.03 & Owen 69 & 23 \\
\hline 4.44 & 10.0 & -1.15 & -0.07 & Ghidini 82 & 9 \\
\hline 4.84 & 12.0 & -1.46 & -0.03 & Jacholkowski 77 & 8 \\
\hline 5.16 & 13.7 & -0.18 & 0.02 & Owen 69 & 23 \\
\hline 5.56 & 16.0 & -0.73 & -0.09 & Anderson 68 & 56 \\
\hline 5.60 & 16.3 & -0.17 & 0.00 & Owen 69 & 23 \\
\hline 7.56 & 30.0 & -0.38 & 0.00 & Baker 83 & 10 \\
\hline 9.73 & 50.0 & -0.44 & -0.00 & Baker 83 & 10 \\
\hline 11.50 & 70.0 & -0.25 & -0.01 & Baker 83 & 10 \\
\hline 13.00 & 90.0 & -0.25 & -0.01 & Baker 83 & 10 \\
\hline
\end{tabular}

Table 7. References to polarization asymmetry $P$ data for $\pi N$ backward scattering. Note that only data for $\sqrt{s}>3 \mathrm{GeV}$ were included in our fit.

\begin{tabular}{|l|c|c|r|r|l|l|}
\hline & $\begin{array}{c}\sqrt{s} \\
\mathrm{GeV}\end{array}$ & $\begin{array}{c}p_{\pi} \\
\mathrm{GeV}\end{array}$ & $\begin{array}{r}u_{\min } \\
\mathrm{GeV}^{2}\end{array}$ & $\begin{array}{r}u_{\max } \\
\mathrm{GeV}^{2}\end{array}$ & Experiment & Ref. \\
\hline$\pi^{+} p$ & 2.40 & 2.59 & -1.40 & 0.03 & Martin 75 & {$[57$} \\
$\pi^{+} p$ & 2.43 & 2.65 & -1.31 & 0.03 & Martin 75 & {$[\underline{57}$} \\
$\pi^{-} p$ & 2.45 & 2.71 & -1.00 & 0.05 & Fukushi. 80 & {$[\underline{50}$} \\
$\pi^{+} p$ & 2.46 & 2.75 & -0.41 & 0.11 & Sherden 70 & {$[49$} \\
$\pi^{+} p$ & 2.53 & 2.93 & -0.44 & 0.10 & Sherden 70 & {$[49$} \\
$\pi^{-} p$ & 2.53 & 2.93 & -0.75 & 0.06 & Sherden 70 & {$[49$} \\
$\pi^{+} p$ & 2.65 & 3.25 & -0.45 & 0.09 & Sherden 70 & {$[49$} \\
$\pi^{-} p$ & 2.65 & 3.25 & -0.77 & 0.06 & Sherden 70 & {$[49$} \\
$\pi^{+} p$ & 2.73 & 3.50 & -0.59 & 0.06 & Bradam. 73 & {$[21$} \\
$\pi^{-} p$ & 2.73 & 3.50 & -0.55 & -0.05 & Fukushi. 80 & {$[50$} \\
$\pi^{-} p$ & 2.73 & 3.50 & -0.89 & -0.23 & Birsa 76 & {$[15$} \\
$\pi^{+} p$ & 2.82 & 3.75 & -0.50 & 0.08 & Sherden 70 & {$[49$} \\
$\pi^{+} p$ & 2.90 & 4.00 & -0.75 & -0.01 & Bradam. 73 & {$[21$} \\
$\pi^{+} p$ & 3.49 & 6.00 & -0.86 & 0.03 & Dick 72 & {$[13$} \\
$\pi^{-} p$ & 3.49 & 6.00 & -0.93 & -0.03 & Dick 73 & {$[14$} \\
\hline
\end{tabular}

\section{References}

1. V. Barger and D. Cline, Phys. Rev. 155, 1792 (1967).

2. V. Barger and D. Cline, Phys. Rev. Lett. 21, 392 (1968).

3. E.L. Berger and G.C. Fox, Nucl. Phys. B 26, 1 (1971).

4. D.T. Gregorich, P. Klaus and R.T. Park, Phys. Rev. D 4, 834 (1971).

5. J.K. Storrow and G.A. Winbow, J. Phys. G 1, 263 (1975).

6. R. Birsa et al., Nucl. Phys. B 122, 397 (1977).

7. C. Baglin et al., Nucl. Phys. B 98, 365 (1975). 
Table 8. References to differential cross section data for $\pi N$ scattering at $\theta=180^{\circ}$. CEX denotes the $\pi^{-} p \rightarrow \pi^{0} n$ charge exchange reaction.

\begin{tabular}{|c|r|r|l|c|}
\hline Reaction & $\begin{array}{r}\sqrt{s}_{\text {min }} \\
\mathrm{GeV}\end{array}$ & $\begin{array}{r}\sqrt{s}_{\text {max }} \\
\mathrm{GeV}\end{array}$ & Experiment & Ref. \\
\hline$\pi^{+} p$ & 3.03 & 3.49 & Lennox 75 & {$[6]$} \\
$\pi^{-} p$ & 3.03 & 3.29 & Kormanyos 66 & 58 \\
$\mathrm{CEX}$ & 3.02 & 3.49 & Kistiakowsky 72 & 59 \\
\hline
\end{tabular}

Table 9. References to differential cross section data for $\pi N$ scattering at $u=0 \mathrm{GeV}^{2}$.

\begin{tabular}{|c|r|r|l|r|}
\hline Reaction & $\begin{array}{r}\sqrt{s}_{\text {min }} \\
\mathrm{GeV}\end{array}$ & $\begin{array}{r}\sqrt{s}_{\text {max }} \\
\mathrm{GeV}\end{array}$ & Experiment & Ref. \\
\hline$\pi^{+} p$ & 3.46 & 5.74 & Owen 69 & {$[23]$} \\
$\pi^{+} p$ & 7.56 & 11.50 & Baker 83 & {$[10]$} \\
$\pi^{-} p$ & 3.75 & 3.75 & Baker 71 & {$[45]$} \\
$\pi^{-} p$ & 3.46 & 5.60 & Owen 69 & {$[23]$} \\
$\pi^{-} p$ & 7.56 & 13.03 & Baker 83 & {$[10]$} \\
$\pi^{-} p$ & 3.99 & 5.56 & Anderson 68 & {$[56]$} \\
$\pi^{-} p$ & 4.22 & 4.84 & Jacholkowski 77 & {$[8]$} \\
$\pi^{-} p$ & 4.44 & 4.43 & Ghidini 82 & {$[9]$} \\
$\pi^{-} p$ & 3.99 & 4.84 & Armstrong 87 & {$[1]$} \\
$\mathrm{CEX}$ & 3.49 & 3.99 & DeMarzo 75 & {$[22]$} \\
\hline
\end{tabular}

8. A. Jacholkowski et al., Nucl. Phys. B 126, 1 (1977).

9. B. Ghidini et al., Nucl. Phys. B 195, 12 (1982).

10. W.F. Baker et al., Phys. Rev. D 27, 1999 (1983).

11. T.A. Armstrong et al., Nucl. Phys. B 284, 643 (1987).

12. H. Aoi et al., phys. Lett. B 35, 90 (1971).

13. L. Dick et al., Nucl. Phys. B 43, 522 (1972).

14. L. Dick et al., Nucl. Phys. B 64, 45 (1973).

15. R. Birsa et al., Nucl. Phys. B 117, 77 (1976).

16. R.T. Park, Phys. Rev. D 10, 3095 (1974).

17. K.L. Mir and J.K. Storrow, J. Phys. G 7, 1187 (1981).

18. J.K. Storrow and G.A. Winbow, Nucl. Phys. B 53, 62 (1973).

19. J. Banaigs et al., Nucl. Phys. B 8, 31 (1968).

20. J. Banaigs et al., Nucl. Phys. B 9, 249 (1969).

21. F. Bradamante et al., Nucl. Phys. B 56, 356 (1973).

22. C. DeMarzo et al., Phys. Lett. B 56, 487 (1975).

23. D.P. Owen et al., Phys. Rev. 181, 1794 (1969).

24. J.P. Boright et al., Phys. Rev. Lett. 24, 964 (1970).

25. A. Donnachie and P.R. Thomas, Nuovo Cim. A 19, 279 (1974).

26. J.F. Gunion, S.J. Brodsky nd R. Blankenbecler, Phys. Lett. B 39, 649 (1972).

27. G. Höhler, Landolt-Börnstein Series, Group I, Elementary Particles, Nuclei and Atoms 9b1, Springer, Berlin, 1983.

28. A.W. Hendry, Phys. Rev. Lett. 41, 222 (1978).

29. R.E. Cutkosky, C.P. Forsyth, R.E. Hendrick and R.L. Kelly Phys. Rev. D 20, 2839 (1979).

30. R. Koch and E. Pietarinen, Nucl. Phys. A 336, 331 (1980).

31. J.K. Storrow, Nucl. Phys. B 47, 174 (1972).

32. K.L. Mir and J.K. Storrow, Z. Phys. C 18, 47 (1983).

33. L.Ya. Glozman, Phys. Lett. B 541, 115 (2002).
34. J.L. Goity and N. Matagne, Phys. Lett. B 655, 223 (2007).

35. S.W. MacDowell, Phys. Rev. 116, 774 (1959).

36. V.N. Gribov, JETP 16, 1080 (1963).

37. J. Schneider et al., Phys. Rev. Lett. 23, 1068 (1969).

38. R.C. Chase et al., Phys. Rev. D 2, 2588 (1970).

39. H. Brody et al., Phys. Rev. Lett. 16, 828 (1966).

40. A. Bashian et al., Phys. Rev. D 9, 3193 (1974).

41. A. Ashmore et al., Phys. Rev. Lett. 19, 460 (1967).

42. A. Babaev et al., Phys. Lett. B 38, 342 (1972).

43. T. Buran et al., Nucl. Phys. B 111, 1 (1976).

44. A. Babaev et al., Phys. Lett. B 67, 351 (1977).

45. W.F. Baker et al., Nucl. Phys. B 25, 385 (1971).

46. A.J. Lennox et al., Phys. Rev. D 11, 1777 (1975).

47. E. Ma and G.L. Shaw, Phys. Rev. D 133027 (1976).

48. A.W. Hendry, Ann. Phys. 136, 1 (1981).

49. D.J. Sherden et. al., Phys. Rev. Lett. 25, 898 (1970).

50. M. Fukushima et. al., Nucl. Phys. B 167, 307 (1980).

51. R.A. Arndt et. al., Phys. Rev. C 69, 035213 (2004).

52. C. Amsler et al. (Particle Data Group), Phys. Let. B 667, 1 (2008).

53. E. Klempt and J.M. Richard, arXiv:0901.2055 [hep-ph], to appear in Rev. Mod. Phys.

54. W.R. Frisken et al., Phys. Rev. Lett. 15, 313 (1965).

55. J. Orear et al., Phys. Rev. 152, 1162 (1966).

56. E.W. Anderson et al., Phys. Rev. Lett. 20, 1529 (1968).

57. J.F. Martin et. al., Nucl. Phys. B 89, 253 (1975).

58. S.W. Kormanyos et al., Phys. Rev. Lett. 16, 709 (1966).

59. V. Kistiakowsky et al., Phys. Rev. D 6, 1882 (1972). 